\title{
The sandwich sign
}

\section{N Mahomed, MB BCh, FCRad (D) H Moodley, MB ChB}

Department of Radiology, University of the Witwatersrand, Johannesburg
The sandwich sign refers to the sandwiching of mesenteric vessels and fat by enlarged mesenteric nodes on cross-sectional imaging, commonly occurring in lymphoma, but not specific to lymphoma. The sign is radiologically indistinguishable from post-transplant lymphoproliferative disorders. The radiological significance of the sandwich sign is in suggesting the diagnosis of lymphoma so that appropriate treatment may be initiated early as the tumour has a rapid growth pattern.

\section{S Afr J Rad 2012;16(3):114-115. DOI:10.7196/SAJR.721}

The sandwich sign is demonstrated on cross-sectional imaging, commonly on CT or ultrasound. It refers to homogeneous softtissue masses representing mesenteric lymphadenopathy as the two halves of a sandwich bun, encasing the mesenteric fat and tubular mesenteric vessels that constitute the 'sandwich filling' (Figs 1 and 2). ${ }^{1,2}$ As the mesenteric lymphadenopathy enlarges, it may compress the mesenteric vessels, partially obstructing venous return, resulting in engorgement of the enhanced mesenteric veins and making them more prominent within the sandwich filling. ${ }^{1}$ Oral and intravenous contrast on abdominal CT scans make the sandwich filling more conspicuous, whereas colour Doppler of the mesenteric vessels highlights the sandwich filling on abdominal ultrasound. ${ }^{1}$

According to the literature, the sandwich sign was previously accepted as specific for lymphomatous mesenteric involvement, with most mesenteric lymphomas being non-Hodgkins lymphoma, but also including post-transplant lymphoproliferative disorders. NonHodgkin's lymphoma cannot be distinguished from post-transplant lymphoproliferative disorders on morphological grounds, as both conditions are caused by the Epstein-Barr virus. ${ }^{1,3}$ It was generally held that - unlike the myriad other causes of mesenteric lymphadenopathy including metastatic carcinoma, sarcoma, carcinoid, AIDS-related lymphadenopathy, Whipple's disease and $\mathrm{TB}$ - only mesenteric lymphoma results in bulky lymphadenopathy that produces the sandwich sign. ${ }^{1}$ This was postulated on the basis of lymphoma's rapid growth without producing clinical symptoms, and the lack of early bowel invasion, perforation or haemorrhage, as frequently seen in some of the above-mentioned tumours. ${ }^{1}$ Inflammatory and infectious aetiologies do not commonly produce such large nodal masses and usually have the appearance of necrosis or rim enhancement, as typically seen in TB. ${ }^{1}$ This theory was refuted by a single study in 2008 by Lien et al. which documents biopsy-proven non-lymphomatous causes of the sandwich sign. ${ }^{4}$ The authors reported that in addition to lymphoma being the most common cause of the sandwich sign, other malignancies such as desmoid tumour, metastatic carcinoma, Mycobacterium avium complex

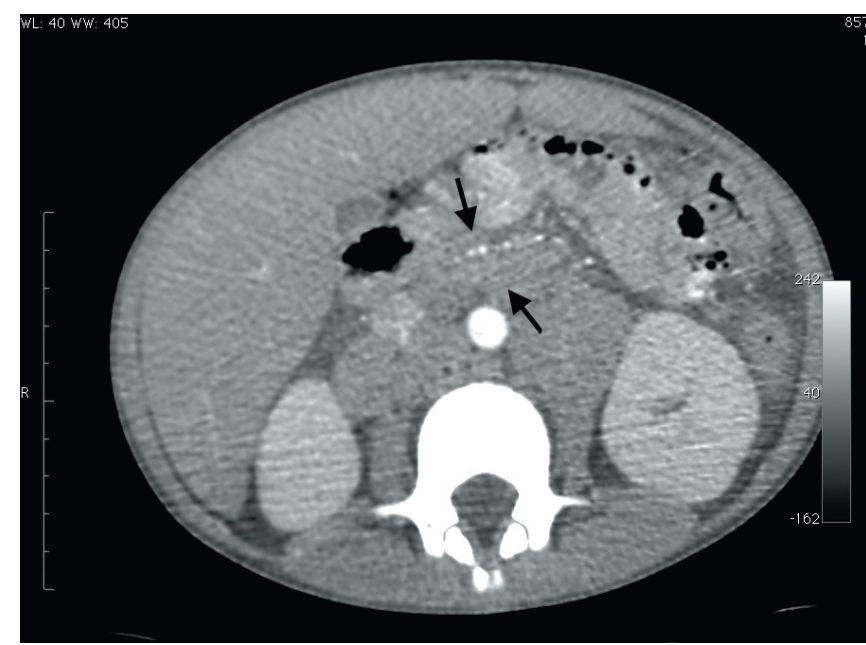

Fig. 1. Axial CT image with intravenous contrast in a 3-year-old diagnosed with non-Hodgkin's lymphoma shows 2 large mesenteric lymphomatous masses (arrows), which represent the halves of the sandwich bun, enveloping mesenteric fat and enhanced vessels. There is also extensive retroperitoneal lymphadenopathy present.

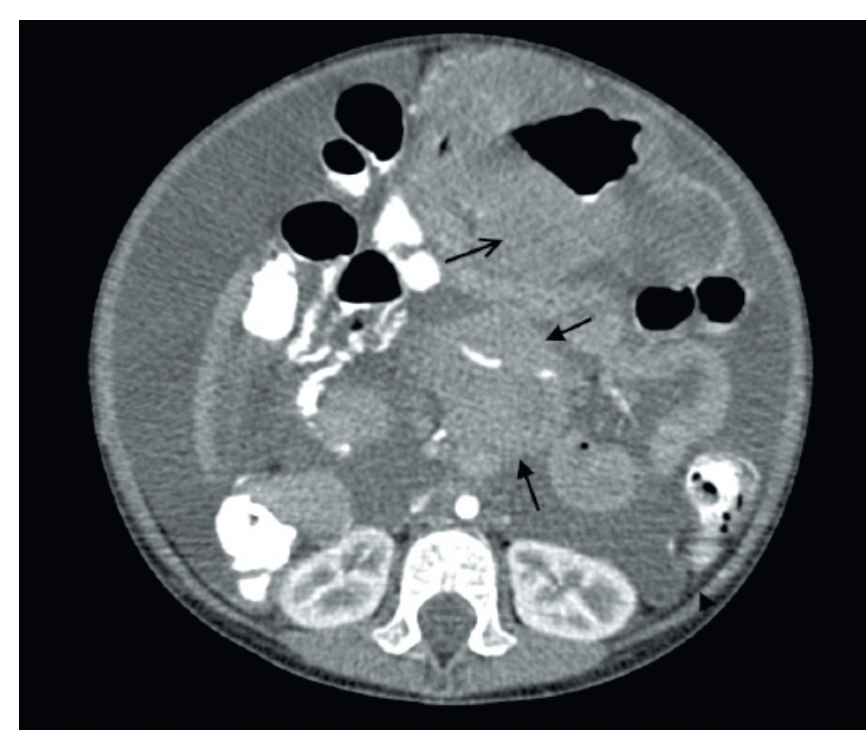

Fig. 2. Axial CT image with intravenous contrast in a 2-year-old girl diagnosed with Burkitt's lymphoma demonstrates the sandwich sign (filled arrows). Note the diffuse lymphomatous infiltration of the stomach (open arrow) and ascites.

(MAC) and TB also demonstrate this sign. ${ }^{4}$ Non-Hodgkin's lymphoma was seen in $50 \%$ and metastatic carcinomas in $41 \%$ of cases. ${ }^{4}$

The classic sandwich sign has evolved to encompass other regions and pathology. Kunimasa et al. have coined a 'thoracic sandwich sign' in reference to anterior-mediastinal lymphadenopathy encompassing the 


\section{SIGNS}

brachiocephalic vein on chest $\mathrm{CT}$ in a patient with T-cell lymphoblastic lymphoma. ${ }^{5}$ The sandwich sign has also been used in the description of traumatic herniation of the stomach on CT, where two semi-circular contrast-filled loops of stomach were separated by a soft-tissue band, indicating that the stomach had folded on itself owing to herniation through a diaphragmatic defect. ${ }^{6}$

In conclusion: the sandwich sign refers to the sandwiching of mesenteric vessels and fat by enlarged mesenteric nodes on crosssectional imaging, commonly occurring in lymphoma, but not specific to lymphoma. The sign is radiologically indistinguishable from posttransplant lymphoproliferative disorders. The radiological significance of the sandwich sign is in suggesting the diagnosis of lymphoma so that appropriate treatment may be initiated early, as the tumour has a rapid growth pattern.

1. Hardy SM. Signs in imaging. Radiology 2003;226:651-652.

2. Toma P, Granata C, Rossio A, et al. Multimodality imaging of Hodgkin disease and non-Hodgkin lymphoma in children. Radiographics 2007;27:1335-1354

3. Ferry JA. Burkitt's lymphoma: Clinicopathologic features and differential diagnosis. Oncologist2006;11:375-383.

4. Lien W, Huang S, Liu K, et al. The sandwich sign of non lymphomatous origin. J Clin Ultrasound 2008;37:212-214.

5. Kunimasa K, Jo T, Takaiwa T, Ishida T. Thoracic sandwich sign. Intern Med 2011;50:2865.

6. Caceres J, Mata JM, Castaner E, Villanueva A. CT recognition of traumatic herniation of stomach: the sandwich sign. J Thorac Imaging 1995;10:150-152. 